\title{
Design of DAODV MANET Routing Protocol
}

\author{
G. Ilanchezhiapandian \\ Research Scholar \\ Department of CSE, \\ Sathyabama University, Chennai
}

\author{
Dr.P. Sheik Abdul Khader \\ Professor \& HOD \\ Department of Computer Applications \\ B.S.Abdur Rahman University, India
}

\begin{abstract}
Ad-hoc networking is a concept in computer communications, which means that users wanting to communicate with each other form a temporary network, without any form of centralized administration. Each node participating in the network acts both as a host and as a router. The study of distributed network and mobile ad hoc network (MANET) are currently two hotspots in distributed computing and mobile communication researching domain. We build up a distributed overlay network on top of MANET's physical infrastructure, to effectively integrate distributed network's advantage on sustaining highly dynamic network into the design of MANET routing protocol. We deploy passive MANET routing algorithms with innovative distributed routing mechanisms. We propose a new kind of MANET routing protocol named distributed Computing based ad-hoc on demand routing (DAODV). Our work indicates that DAODV has an improved routing performance in comparison with the popularly used AODV protocol.
\end{abstract}

\section{Keywords}

MANET, DAODV, distributed network.

\section{INTRODUCTION}

In ad-hoc networks, communications are done over wireless media between stations directly in a distributed fashion without the help of wired base station or access points. With the popularity of ad-hoc networks, many routing protocols have been designed for route discovery and route maintenance. They are mostly designed for best effort transmission without any guarantee of quality of transmissions. Some of the most famous routing protocols are Dynamic Source Routing (DSR) Ad-hoc On Demand Vector (AODV) Optimized Link State Routing protocol (OLSR), and Zone Routing Protocol (ZRP). On the other side, Distributed network is currently a hotspot in the international network researching domain. Different kinds of distributed network models such as Napster, Freenet and Gnutella have already been popularly used. However, each of them has some open problems [2], [3]. To solve these problems, structured DISTRIBUTED overlay networks (SON) such as CAN, Chord [4], Pastry and Tapestry are proposed to provide a kind of mechanism to improve network scalability [5]. All of these SON algorithms have a common ground that they all assign keys to data and nodes through a hashing function, and at the same time they use these keys to store and maintain data which are shared in the network [6].

\section{OVERVIEW OF MANET AND DISTRIBUTED NETWORK}

DISTRIBUTED networks are popular Internet applications that enable users to share computing resources. Today, DISTRIBUTED, Web browsing, and email can be put together in the same class of importance. Napster is the tool probably most responsible for the popularization of DISTRIBUTED networks due to its music sharing support. So far, several Napster clones and general file sharing systems have been created, such as KaZaA, BitTorrent, and eMule. DISTRIBUTED networks are composed of software nodes (peers) installed on the edges of the Internet, usually in inexpensive user hosts along diverse administrative domains. Each peer communicates with other peers directly or indirectly to share local resources (e.g., memory, files, processing power), forming robust distributed systems over the Internet. The DISTRIBUTED communication model clearly differs from the client-server model. In client-server, servers store resources accessed by clients, by contrast, in DISTRIBUTED each peer is itself a repository that shares its resources with other peers, thus forming environments where resources are distributed among peers acting as both servers and clients.

Client-server addressing is based on the Internet Domain Name System (DNS), while DISTRIBUTED networks employ addressing schemes independent from DNS to index peers, users, and resources. Client-server messages are routed at the network layer by Internet routers, but DISTRIBUTED messages are routed at the application layer by routing peers.

Having routing and addressing built on top of Internet protocols, DISTRIBUTED networks are quite dynamic environments where new peers can join the network when they are started in user hosts, and leave when users shutdown the peers. This dynamic nature makes DISTRIBUTED networks both unpredictable and scalable: unpredictable because resources may be unavailable if the peers that host them are down; scalable because the expansion of the network capacity requires only the addition of new peers.

During the last couple of years tremendous improvements are made in the research of ad hoc networks. Due to its possibility to create and organize a network without any central management, MANET is characterized as the art of networking without a network [7]. Currently, the research of DISTRIBUTED network and MANET are always taken as two separated areas. But the following similarities are seen between these two technologies (see Figure 1).

\subsection{Dynamic Network Topology}

Nodes in DISTRIBUTED networks randomly join and leave the network, which causes the network topology to change frequently with time. It is the same with MANET. And the mobility character of mobile nodes in MANET can make network topology changes much more frequent [8]. 


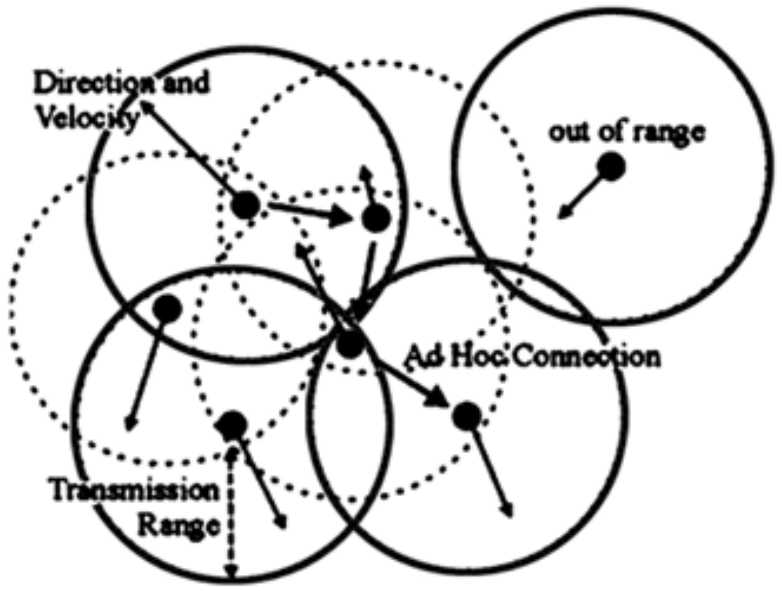

(a) Architecture of MANET

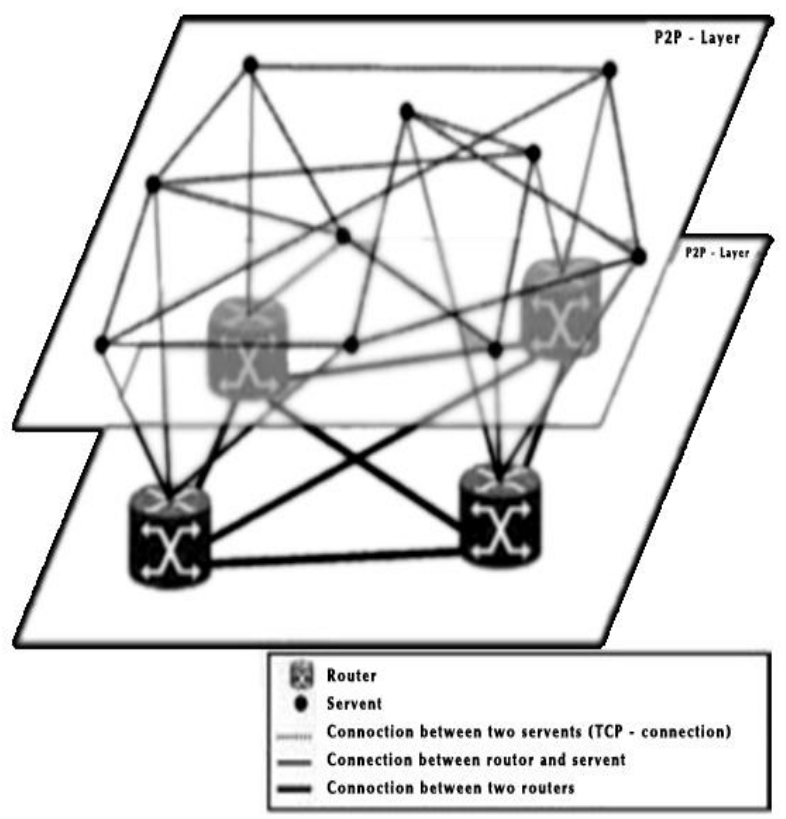

(b). Architecture of DISTRIBUTED Network

Fig. 1 Similarities of MANET and DISTRIBUTED Networks

\subsection{Multi-hop Connection}

Nodes in both MANET and distributed networks connect with each other via multi-hop routing. Hop-to-hop connections in DISTRIBUTED networks are via TCP links with physically unlimited range, while hop-to-hop connections in MANET are via wireless links, limited by the radio transmission range.

\subsection{Decentralized control}

Both DISTRIBUTED network and MANET have a decentralized structure. There is no central administration as what is seen in the Client/Server network model. Nodes in the network are all peers and can communicate directly to each other. The network will not suffer the paralysis and bottleneck caused at the central server, which improves the robustness of the network.

\subsection{Multi-Identity of Nodes}

Nodes in DISTRIBUTED network and MANET act both as a client and a server, as well as a router. Each node can route and transfer messages independently. And they can also provide resource downloading and communicate with each other directly [9].

\section{DESIGN OF DAODV PROTOCOL 3.1 Architecture of DAODV Model}

The architecture of the DAODV model is based on DISTRIBUTED computing technology. Figure 2 shows that DAODV protocol is integrated with the DISTRIBUTED overlay network which is built upon the physical network of the MANET.

\begin{tabular}{|c|c|c|c|}
\hline $\begin{array}{c}\text { File } \\
\text { Discovery } \\
\text { Service }\end{array}$ & $\begin{array}{c}\text { Information } \\
\text { Administration } \\
\text { Service }\end{array}$ & $\begin{array}{c}\text { File } \\
\text { Transfer } \\
\text { Service }\end{array}$ & $\begin{array}{c}\text { Communication } \\
\text { Security Service }\end{array}$ \\
\hline \multicolumn{3}{|c|}{ DISTRIBUTED Computing Based MANET Routing } \\
Algorithm \\
\hline \multicolumn{3}{|c|}{ DISTRIBUTED Overlay Network (Node Naming and } \\
Indexing) \\
\hline
\end{tabular}

Fig. 2 Architecture of DAODV Model

\subsection{Basic Design}

DAODV uses the node naming mechanism used in Chord. It adopts a consistent hashing algorithm [10], [11]. Each node is assigned an m-bit node identifier (NID), which is obtained by hashing the node's IP address. Thus we are able to specify the location of the node in the hash ring. When a node joins the network for the first time, a new NID ranging from 0 to $(2 \mathrm{~m}-1)$ is automatically assigned based on the adopted hashing algorithm. To simplify the routing algorithm, we have adopted the discontinuous naming mechanism used in Chord. The node state is maintained in the DAODV routing table (PRT) which is similar to the routing table of AODV. The difference is that, DAODV uses the NID for the destination node and the next hop, whereas the later uses the node's IP address.

\subsection{Route discovery Algorithm}

The DAODV route discovery algorithm discovers the route from the source node to the destination node reactively. The route discovery is triggered by the mobile nodes under any of the following, and an update is required in the PRT table.

i. A node initiates the route discovery procedure.

ii. When a node receives the route query message (RREQ), it queries the PRT, if the route is null or out dated, the route discovery method is invoked.

iii. When a route issues a route error message (RERR) to the node which initiated the data transmission. The initiating node starts the route query method. 
A description of the route discovery algorithm is given below:

1) Node $M$ checks for RREQ in cache. If the source identifier and the request identifier is the same, the request is discarded.

2) $M$ checks in the Route Record whether RREQ message contains NID(M). If found, the message is discarded.

3) $M$ checks whether the destination node is present in the PRT entries. If found go to step 5, otherwise to step 6.

4) $M$ checks whether the destination node is $M$ itself. If true, go to step 5, otherwise to step 6.

5) $\mathrm{M}$ checks whether the route is bi-directional. If it is, the route reply message (RREP) is send to the initiating node $S$ directly. Otherwise, the route discovery method is invoked.

6) $M$ adds itself to the route record and broadcasts RREQ message to the neighbors.

\subsection{PRT Querying Algorithm}

The PRT querying algorithm queries the PRT and picks up the Next Hop Ring Interval (NRI) which is the closest to D. Any outdated entries are updated. The algorithm is depicted as follows:

1) When a node $M$ initiates or receives a RREQ message, it compares the destination node identifier of the RREQ with $\mathrm{NID}(\mathrm{M})$. If they are matched, $\mathrm{M}$ will return RREP to querying initiating node $\mathrm{S}$.

2) $M$ will search into the PRT and compares the destination node identifier (D) with the PRT entries to find the NRI to which D belongs for message transfer.

3) $\mathrm{M}$ triggers the route discovery for the destination node and waits for the response. If the response if received the PRT is updated, otherwise a failure message is send.

\subsection{DAODV Route Update and Maintenance}

Route maintenance is required due to the nodes' mobility. In MANETs, a node's position changes with time, this leads to dynamic network topology. A destination node may become out of reach due to normal or abnormal departure or has moved out of the transmission range. When this occurs, the route updating algorithm is triggered reactively.

When there is a failure in the data transmission, the node which initiated the packet transfer will issue an overdue message to the related nodes. Thus the PRT entries are outdated and the route update is triggered. If the route discovery process ends in a failure, it means that the destination node has moved out of range. Therefore the destination node is marked as invalid.

Due to the high dynamic character, mobile nodes may join and leave the network at any time. To save the route maintenance cost, when a node joins the network, DAODV will not initialize its PRT. On the contrary, the PRT is constructed only on demand.

DAODV nodes will only overhear the source route related to the next hop node in its local PRT. By doing this, DAODV inherits all the advantages of AODV at the same time limits. By the use of Phello messages in DAODV, mobile nodes broadcast phello messages reactively.
Therefore the information about the neighbor nodes are collected only when there is a need for route discovery. If the destination node is one of the neighbors, the route discovery process stops and RREP is returned to the source. Consequently, the overload of nodes in DAODV is reduced

\section{SIMULATION RESULTS}

Simulation is done with fifty nodes in a $1000 \mathrm{~m} * 1000 \mathrm{~m}$ space. The nodes are randomly distributed in the simulation scope. And each node performs a Brown movement during the simulation period. The traditional pause time (PT) is used to define the mobile character of the model. At the beginning of simulation, a node keeps static for a PT. Then it will randomly choose a destination node and moves toward it with a certain speed. When it arrives, the node will keep static for a PT and then choose a node as the next destination.

This process will be repeated till the simulation ends. We choose for different values of PT. To simulate the network services' influence on DAODV's performance, we randomly choose 20 pairs of nodes to transmit CBR packets to each other. The CBR packets are 128 bytes packets with a transmission rate of 4 packets per second

\subsection{Packet Deliver Ratio}

Packet deliver ratio is an important criterion in estimating a routing protocol's performance in how well it adapts to the change of network topology and how efficient it provides a route discovery. Fig 3 depicts the .

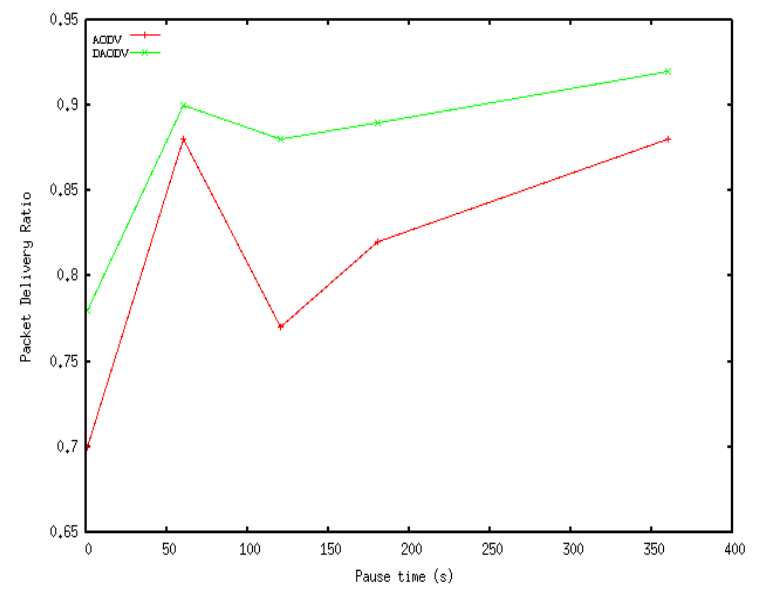

Fig. 3 Comparison of PDR between AODV and DAODV

The probability of link failure between nodes and route failure decreases when the mobility of nodes decreases. The success rate and stability of packet transmission in DAODV is much better than AODV. The reason is that DAODV has improved the node discovery and packet delivery performance by taking advantages of the structured DISTRIBUTED routing mechanisms. However, the caching mechanism adopted in AODV is the origin of its problem in providing out-of-time network status and its low packet deliver ratio.

\subsection{Average end to End Delay (AED)}

Average end-to-end delay estimates how efficient and fast route discovery is done. Figure 4 depicts the comparison of Average End-to-End Delay between DAODV and AODV with Pause Time. 


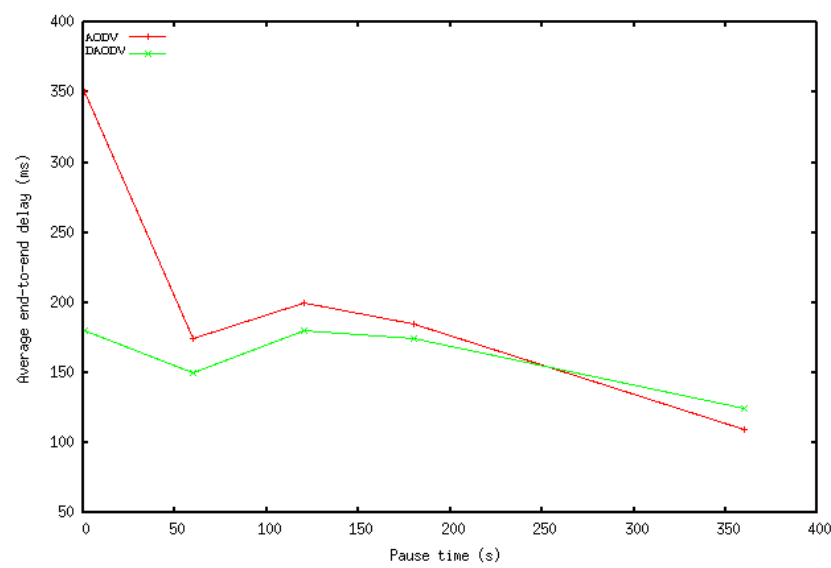

Fig. 4 Comparison of AED between AODV and DAODV

AODV employs broadcasting based route discovery mechanism and consumes longer time to re-discover a route when the current path has failed. On the contrary, DAODV improves the route discovery efficiently by building up a DISTRIBUTED overlay network on MANET's physical topology and introducing PRT to accelerate the route discovery process.

\subsection{Node Storage Overhead (NSO)}

Figure 5 depicts that DAODV effectively decreases the route storage overhead for each node, and at the same time it decreases the route discovery and maintenance overhead related to these cached routes as well.

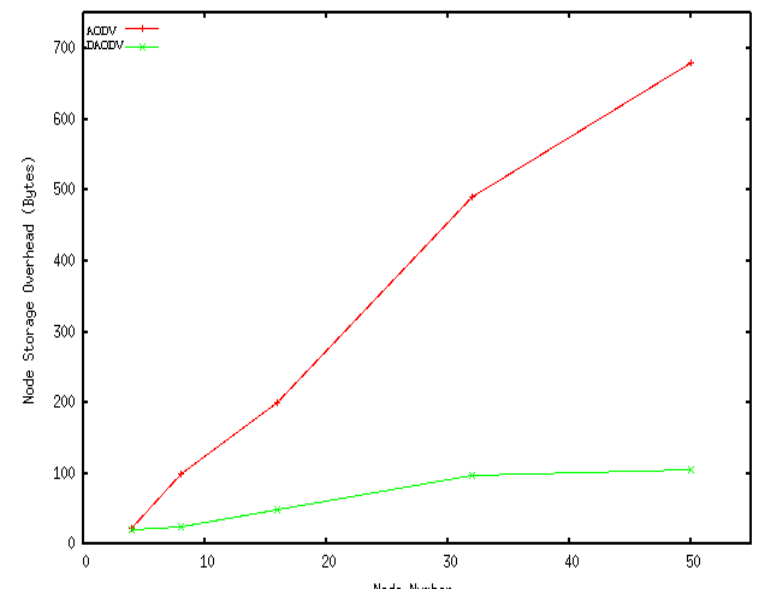

Fig. 5 Comparison of NSO between AODV and DAODV

The cached routes for a mobile node in AODV are in direct proportion to the number of nodes in the network. That is, for a MANET with $\mathrm{N}$ nodes, the cached routes for each node is $\mathrm{O}(\mathrm{N})$. On the contrary, by implementing structured DISTRIBUTED routing mechanism, the relationship of cached routes for each node in DAODV with the number of nodes is $\mathrm{O}(\log \mathrm{N})$. By doing this, DAODV effectively decreases the route storage overhead for each node, and at the same time it decreases the route discovery and maintenance overhead related to these cached routes as well.

\subsection{Routing Overhead (RO)}

Figure 6 depicts the comparison of Routing Overhead between DAODV and AODV with Pause Time.

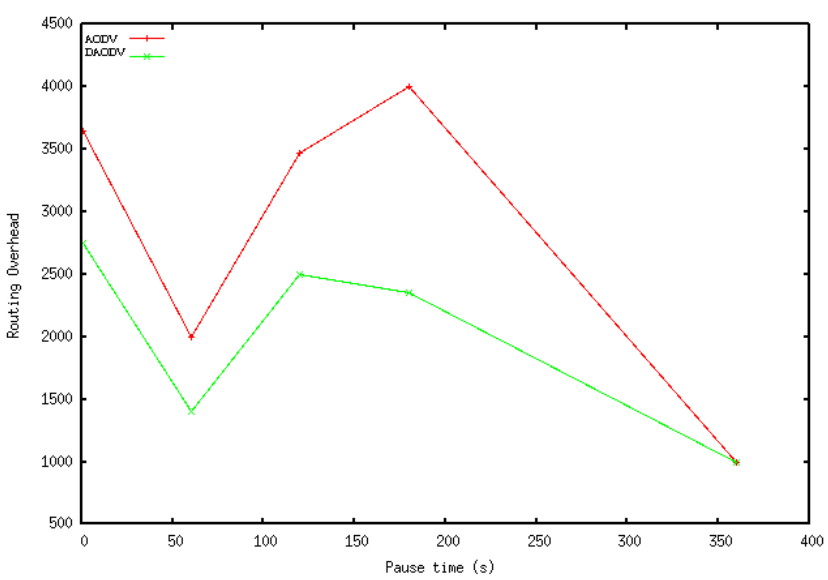

Fig. 6 Comparison of RO between AODV and DAODV

The number of route packets in AODV is huge due to the number of cached routes for each node is huge. The route maintenance and update processes caused by these cached routes consume much of the network bandwidth and decrease the performance of AODV. Whereas DAODV improves the routing performance by introducing structured DISTRIBUTED overlay network technology and limiting the number of cached routes.

\section{CONCLUSION}

DAODV seamlessly integrates the functions of DISTRIBUTED overlay routing protocols operating in a logical namespace with those of MANET routing protocols operating in a physical namespace. By this means, DAODV has inherited all the advantages of DISTRIBUTED networking technology in sustaining highly dynamic networks and maintaining network scalability. Besides these, DAODV introduces a series of optimizing mechanisms to solve the detouring problem of structured DISTRIBUTED overlay network and improves routing efficiency.

\section{REFERENCES}

[1] Mengkun Yang and Zongminm Fei, "A novel approach to improving search efficiency in unstructured peer-to-peer networks," Journal of Parallel and Distributed Computing, Volume 69 Issue 11, November, 2009

[2] J. Xu, "On the fundamental tradeoffs between routing table size and network diameter in peer-to-peer networks," IEEE INFOCOM, 2003 - The Conference on Computer Communications, Vol. 22, No. 1, Mar 2003, pp. 2177 2187.

[3] S. Sen and J. Wang, "Analyzing peer-to-peer traffic across large networks," IEEE/ACM Transactions on Networking, Vol. 12, No. 2, Apr 2004, pp. 219-232.

[4] I. Stoica, R. Morris, D. Liben-Nowell, D. R. Karger, M. F. Kaashoek, F. Dabek, and H. Balakrishnan, "Chord: A scalable peer-to-peer lookup protocol for internet applications," IEEE/ACM Transactions on Networking, Vol. 11, No. 1, Feb 2003, pp. 17-32. 
[5] J. Xu, A. Kumar, and X. Yu, "On the fundamental tradeoffs between routing table size and network diameter in peerto-peer networks," IEEE Journal on Selected Areas in Communications, Vol. 22, No. 1, Jan 2004, pp. 151-163.

[6] S. Jain, R. Mahajan, and D. Wetherall, "A study of the performance potential of DHT-based overlays," 4thUSENIX Symposium on Internet Technologies and Systems(USITS 2003), March 2003.

[7] S. Jiang, Y. Liu, Y. Jiang, and Q. Yin, "Provisioning of adaptability to variable topologies for routing schemes in MANETs," IEEE Journal on Selected Areas in Communications, Vol. 22, No. 7, Sep 2004, pp. 13471356.

[8] D. Cavalcanti, D. Agrawal, C. Cordeiro, B. Xie, and A. Kumar, "Issues in integrating cellular networks, WLANs, and MANETs: A futuristic heterogeneous wireless network," IEEE Wireless Communications, Vol. 12, No. 3, June 2005
[9] C. H. Foh and B. S. Lee, "A closed form network connectivity formula for one-dimensional MANETs," ICC 2004 - IEEE International Conference on Communications, Vol. 27, No. 1, June 2004, pp. 37393742 .

[10] A. Duran and C.-C. Shen, "Mobile ad hoc P2P file sharing," WCNC 2004 - IEEE Wireless Communications and Networking Conference, Vol. 5, No. 1, March 2004, pp. 114-119.

[11] J. Mischke and B. Stiller, "Efficient protocol specification and implementation for a highly scalable peer-to-peer search infrastructure," NOMS 2004 - IEEE/IFIP Network Operations and Management Symposium, Vol. 9, No. 1, Apr 2004, pp. 411-424. 